\title{
Locating Identities in Time: An Examination of the Formation and Impact of Temporality on Presentations of the Self through Location-Based Social Networks
} \author{
Institute of Technology \\ IOANNA LYKOURENTZOU, Utrecht University \\ VASSILIS-JAVED KHAN, Eindhoven University of Technology \\ ALAN CHAMBERLAIN, University of Nottingham \\ TING CAO, Rochester Institute of Technology \\ MICHAEL SAKER, City, University of London \\ NICOLAS LALONE, University of Nebraska at Omaha
}

KONSTANTINOS PAPANGELIS, Niantic x RIT Geo Games and Media Research Lab, Rochester

\begin{abstract}
Studies of identity and location-based social networks (LBSN) have tended to focus on the performative aspects associated with marking one's location. Yet these studies often present this practice as being an a priori aspect of locative media. What is missing from this research is a more granular understanding of how this process develops over time. Accordingly, we focus on the first 6 weeks of 42 users beginning to use an LBSN we designed and named GeoMoments. Through our analysis of our users' activities, we contribute to understanding identity and LBSN in two distinct ways. First, we show how LBSN users develop and perform self-identity over time. Second, we highlight the extent these temporal processes reshape the behaviors of users. Overall, our results illustrate that although a performative use of GeoMoments does evolve, this development does not occur in a vacuum. Rather, it occurs within the dynamic context of everyday life, which is prompted, conditioned, and mediated by the way the affordances of GeoMoments digitally organize and archive past locational traces.
\end{abstract}

CCS Concepts: • Human-centered computing $\rightarrow$ User studies; Field studies; Ubiquitous and mobile computing;

Additional Key Words and Phrases: Location-based social networks, self-identity, impression management, temporality

Dr A. Chamberlain's part in this work was supported by the Engineering and Physical Sciences Research Council (grant number EP/T51729X/1) projects RCUK Catapult Researchers in Residence award Digital-Disruptive Beats-Music-AICreativity-Composition and Performance, and (grant no. EP/V00784X/1) UKRI Trustworthy Autonomous Systems Hub. Authors' addresses: K. Papangelis, Golisano Hall 2521, Rochester, New York, USA, 14623; email: kpapangelis@me.com; I. Lykourentzou, Utrecht University, Princetonplein 5, Utrecht, The Netherlands, 3584; email: I.Lykourentzou@uu.nl; V.J. Khan, Sappi Europe, 166 Chaussee de la Hulpe, Brussels, Belgium, B-1170; email: V.J.Khan@tue.nl; A. Chamberlain, University of Nottingham. Mixed Reality Lab, Wollaton Road, Nottingham, United Kingdom NG8 1BB; email: Alan.Chamberlain@nottingham.ac.uk; T. Cao, Golisano Hall 3525, Rochester, New York, USA, 14623; email: anita_cao@live.com; M. Saker, City, University of London, Northampton Square, London, United Kingdom, EC1V 0HB; email: Michael.Saker@city.ac.uk; N. LaLone, University of Nebraska at Omaha, PKI 174i , 6001 Dodge St, Omaha, Nebraska, USA, 68182.

Permission to make digital or hard copies of part or all of this work for personal or classroom use is granted without fee provided that copies are not made or distributed for profit or commercial advantage and that copies bear this notice and the full citation on the first page. Copyrights for third-party components of this work must be honored. For all other uses, contact the owner/author(s).

(C) 2021 Copyright held by the owner/author(s).

2469-7818/2021/10-ART10 \$15.00

https://doi.org/10.1145/3473043 
ACM Reference format:

Konstantinos Papangelis, Ioanna Lykourentzou, Vassilis-Javed Khan, Alan Chamberlain, Ting Cao, Michael Saker, and Nicolas LaLone. 2021. Locating Identities in Time: An Examination of the Formation and Impact of Temporality on Presentations of the Self through Location-Based Social Networks. ACM Trans. Soc. Comput. 4, 3, Article 10 (October 2021), 23 pages.

https://doi.org/10.1145/3473043

\section{INTRODUCTION}

The transition to a mobile web that incorporates the global positioning system (GPS) has provided the necessary conditions for location-based social networks (LBSNs) to emerge. The subsequent overlaying of physical environments with digital information produces "hybrid space," which users can access with their smartphones as they move through their physical environments [27]. A hybrid space affords individuals the ability to form social networks pertaining to their location in the physical world through location-tagged media content, such as photos, video, and text [70]. Notable examples of LBSNs include Foursquare, ${ }^{1}$ Glympse, ${ }^{2}$ and, more recently, Swarm. ${ }^{3}$

Following the ascent of locative media, a significant body of research has gradually built around locative applications. For Saker and Evans [57], research in the field has predominately focused on the ability of locative applications to coordinate social interactions in space and place $[34,41,52,69]$, increasing the sense of co-presence [12, 49], altering how people approach their physical environment [41, 42, 67], turning everyday life into a game [20, 30, 36, 54], and reshaping how mobile media is understood [10, 11, 61]. And as Saker and Evans [27] similarly note, studies on locative media have revolved around the more performative aspects of LBSN use, which has implications for how users might present themselves through these applications.

Studies examining self-identity and LBSN have concentrated on performance through marking one's location in the context of impression management [26, 35, 58], self-representation and identity [21, 63], friendship performance [4, 8], and reflection and memory [59]. Although self-identity, then, has been the focus of scholastic circumspection, only a handful of studies (e.g., [27, 60]) have touched upon the relationship between locative media and the evolution of self-identity performances and narratives over time. Likewise, studies that do examine the concept do so through theorizations about the topic based on various psycho-socio frameworks (e.g., [27, 59, 60]). What is missing from these discussions is a more granular examination of how time-based evolution or temporality ${ }^{4}$ affects self-identity performances "in the wild" over a specific period. Addressing this connection is important because it can help us better understand the implications of LBSN usage and what influences user connections with them over time.

Through a study of 42 users over a period of 6 weeks, our research builds upon previous work [51] and contributes to the understanding of temporality and LBSNs. It does this by providing two things. First, our study provides an understanding of (1) how self-identity forms in LBSN and how it impacts LBSN use over time, and (2) that users comprehend temporality as being an explicit part of their "self" and as such go to great lengths to curate it as their LBSN self-identity changes over time. Next, our study provides an affirmation that temporality is not only a central concept in understanding LBSN use, but also that (1) it is intertwined with the performative nature of

\footnotetext{
${ }^{1}$ https://foursquare.com/.

${ }^{2}$ https://glympse.com/.

${ }^{3}$ https://www.swarmapp.com/.

${ }^{4}$ The term temporality is used in this article to refer to the phenomenological experience of living within time that encompasses the past, present, and future.
} 
user-to-user and user-to-place interactions, and that (2) it can prompt the activation of the user contribution to the LBSN and instigate interaction across users.

These contributions manifest through an examination of user behavior in GeoMoments, an LBSN we designed and developed, and contribute to the models of locative media and identities by adding a "temporal" dimension. It should be mentioned that to the best of our knowledge, the present study is the first study that touches on the topic, as previous studies on the topic have explored self-identity in the context of LBSN utilizing one-off approaches to explore self-identity in LBSN, and for the most part, they have not explicitly touched upon the interplay of temporality and self-identity in an LBSN context.

In the following section, we provide a brief but detailed overview of related LBSN literature, describing the works on self-identity, and highlighting the importance of studying how expressions of self through LBSN evolve over time. Following this, we explain our methodology. This begins with an introduction of our LBSN, GeoMoments, before describing how it differs from other LBSNs, as well as how the affordances of our LBSN are commensurate with the underlying research objectives of this work. Moving forward, we then illustrate our findings, elaborating on the different ways that self-identity is manifested throughout our study in the context of LBSN. We conclude with a discussion of our findings, the limitations of this work, and potential future research directions.

\section{BACKGROUND}

Early research examining the interplay between self-identity and LBSN has tended to frame the performative side of LBSN $[19,67]$ using Sutko's and de Souza e Silva's conceptualizations of "presentation of place" and "presentation of location," exploring the experience of being within and belonging to virtual communities, alongside identity formation in online and offline spaces (e.g., [33]). As illuminating as this research has been, it has not sufficiently focused on how users construct their identities within a mixture of physical and digital settings. More recent studies of LBSN and the performance of identity have consequently primarily drawn on Goffman's division between "front stage" and "back stage" behaviors [32].

Goffman uses the theatrical metaphor of "front stage" and "back stage", and defines the individual as an actor, and his social interactions as dramaturgical externalized performances shaped by environment and audience, aimed at creating specific impressions. The result is a series of "masks" that an individual wears according to the social situations. The "masks" according to Goffman is "the positive social value a person effectively claims for himself by the line others assume he has taken during a particular contact" [32].

Goffman's metaphors have been used extensively as analytical frameworks when studying the self-identity and humans' social interactions online as part of LBSN (and more traditional social network sites) as individuals (1) construct a public or semi-public profile within a bounded system, (2) articulate a list of other users with whom they share a connection, (3) view and traverse their list of connections and those made by others within the system, (4) interact with individuals within the system, and (5) produce context for consumption by other individuals [4]. It should be noted that for the most part, the literature that uses Goffman's metaphors as an interpretative framework for exploring self-identity and interactions in social media focuses only on the "front stage" behavior, which is behavior that is externalized in social networks, and not in "back stage" behavior, which is behavior that is not (or cannot be) externalized in social media (e.g., thought process and internal schemas associated with the externalizations) [5, 37, 56].

As a corollary to this, then, and as Saker [58] rightly point out, related research has primarily attended to the performative side of marking one's location through LBSN in the context of impression management $[26,35,58]$, self-representation and identity $[21,63]$, friendship performance 
$[4,8]$, and reflection and memory [59]. What these studies illustrate is that LBSN can contribute new "front stage" ways of performing a particular identity and self-representation narrative as an "on-going project that is constantly being modified, updated and safeguarded" [51].

The motivations for actions (e.g., location disclosure as well as non-disclosure) of individuals through LBSN are imbued with identity-based representations. This is due to the majority of users (if not all) considering their actions in LBSN as part of their presentation of "self" [62]. Accordingly, these actions tend to be highly curated and undertaken to present a certain depiction of the self: the type of places users choose to "check in" to, take photos in, or walk through hold valuable meaning beyond their abstracted physical location [28, 39, 53].

Through Goffman's division of "front stage" and "back stage" or Butler's notion of performativity, Schwartz and Halegoua propose the "spatial self" [62] as a cogent conceptual framework to be used to understand the interplay between identity performance and locative media usage. The spatial self is a theoretical lens that comprehends the presentation of the self as involving geographic traces recorded through locative media. The spatial self refers to and interprets "a variety of instances (both online and offline) where individuals document, archive, and display their experience and/or mobility within space and place in order to represent or perform aspects of their identity to others" [62]. Studies that acknowledge the spatial self, for the most part, discuss self-identity in LBSN in a more lived, fluid, and experiential manner. Importantly, these studies do so by (1) highlighting the dynamic interplay between virtuality, spatiality, and materiality of self-identity and LBSN, (2) by considering LBSN as a device of self-representation, and user activity as the main vehicle of user narratives, and (3) by positioning "embodied LBSN use" as the main catalyst behind behaviors and interactions associated with self-identity performances and narratives in the visible and invisible of the hybrid spaces that are created through LBSN [18].

What this work suggests is that (1) there is a clear interplay between self-identity and activity/actions of individuals, and this has the potential to affect behavior, perception, and interaction in both the virtual and the ordinary [58,62], (2) self-identity narratives and performances through LBSN can affect the polysemic meaning of physical places and have the potential to disrupt hierarchical or hegemonic modes of understanding physical space and place [23, 31, 55], and (3) individuals safeguard their LBSN self and places that hold valuable meaning to them through playful antagonistic behavior that oftentimes manifests through territorial claims [16, 41, 54, 54].

Although the "spatial self" does address spatial connections between locative activities and selfidentity in LBSN usage, this relationship is nonetheless abstracted from a more meaningful understanding of temporality. It does not explicitly address the preservation of temporal features of LBSN usage [59]even though the archival aspect of the spatial self is touched on by Schwartz and Halegoua [62]. The most prominent studies that have attended to this dimension are those of Saker and Evans [27], as well as Drakopoulou [22]. Saker and Evans explored the archival function of the LBSN Foursquare, positing that it effectively enabled the locational past to permeate the present on a daily basis, as well as to forge an orientation to the future that felt more congruent with the locational self thus recorded [27]. Following this vein of thought, Drakopoulou [22] asserts that LBSN with time-based interfaces can be viewed as "durational objects in the sense of presenting a complex relation of past, present, and future as [archived] memories," and that they affect identity by providing a retrospective view of the recent past. This position resonates with Parika [44], who argues that memories supported by digital technologies, such as LBSN, force a rethinking of memory perception and organization. Hoskins refers to this as the connective turn. As he explains, "the formation of memory is increasingly structured by digital networks, [with] memory's constituting agency [being] both technological and human" [38].

Studies that have explored the temporal dimension of self-identity in LBSN have shown that the present (time) can be restructured and reconsidered by the projection of the past into the 
future through locative media [22, 27, 60]. This suggests that the practice of LBSN can potentially configure durational mediated memory objects that organize and archive digitally externalized "memories" [27, 66]. Such an approach allows the interpretation of historical location-based data in terms of their social functions, intentions, context, and situations under which they are produced as self-knowledge that has the potential to modify the self [55].

Moreover, these studies illustrate that identity is thought to be malleable, and that future selfidentity narratives and performances depend on past memories, which are comprised of archived activities enabled through the interface of the LBSN. This results in individuals treating their archived Foursquare check-ins, for example (their geo-coded traces of activity and self-identity narratives), as memories and oftentimes reflect upon them and use them to change their future behavior [60]. This illustrates that memories can become interconnected in ways that were not originally intended while facilitating reflection beyond the extent of the regular users of LBSNs $[22,60]$.

Overall, the literature on self-identity and LBSN illustrates that LBSNs have created novel modes of interaction that have in turn led to new forms of narration, identity formation, and identity maintenance. Although this is the case, only a handful of studies have touched upon temporality and self-identity narratives. Accordingly, within the canon of locative media, there is a need to further address the use of LBSN as it develops and forms over time. Doing so not only has the potential to provide new insights and understandings regarding LBSN use but could also provide meaningful information about how individuals engage, effect, and are affected by LBSN. To this end, our 6-week study of GeoMoments research advances along two lines. First, our research explores how self-identity performances through LBSN form and develop over time. Second, our research examines to what extent this temporal process reshapes the behaviors, practices, and self-reflections of users.

\section{METHODOLOGY}

The present research is focused on performativity in LBSNs. This performance, which we have operationalized through the dramaturgy of Goffman [32], offers us a unique lens through which to understand how self-identity manifests within the context of LBSN. Through this lens, we are able to critically explore and understand the roles and impacts of mobile media. These impacts are manifest through the use and design of public space, urban infrastructure, mobility patterns, local sentiment, and experiences of place $[40,58,62]$. To get at these processes, we created an LBSN called GeoMoments. Our process from beginning to end can be seen in Figure 1. Of note in Figure 1 is the reciprocal nature of our initial research and our prototyping stage. Once those steps were complete, our study, the present research, was able to be performed. This section outlines (1) the design of GeoMoments, (2) our study design, (3) our data collection processes, and (4) how we analyzed our data. To examine self-identity and temporality in LBSNs, we designed, developed, and implemented GeoMoments.

\subsection{GeoMoments}

We developed a bespoke LBSN from scratch (rather than using existing ones) for three reasons. First, our study takes place in a large urban center of China. Mainstream LBSNs (e.g., Foursquare, Swarm) and the mapping services associated with these (e.g., Google Maps) are mostly unavailable or limited in this region. Second, using a custom-made application means it possible to have full control over the design and LBSN variables that need to be gathered. Not only does this set our study apart from extant research on LBSN but it also means we can adapt and modify this LBSN in accordance with the findings of our study for future projects. Finally, working with an LBSN other 


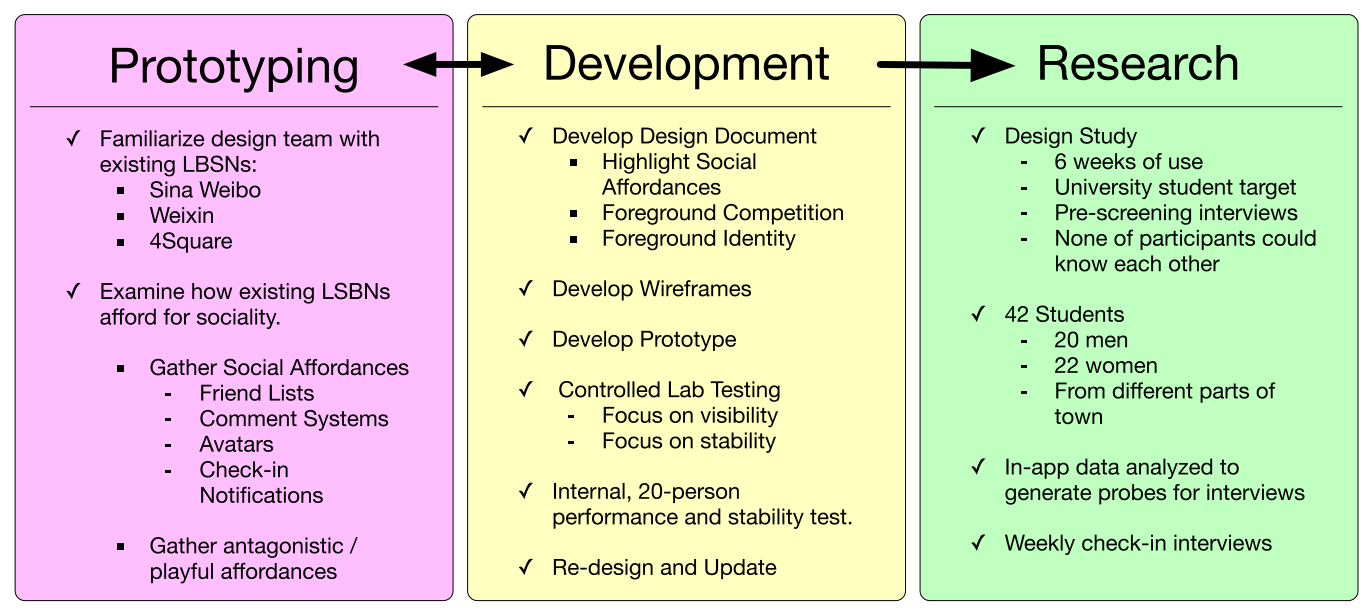

Fig. 1. Our design process began by examining LBSNs and unpacking what aspects of them were relevant to sociality. Next, we developed a design idea and created a prototype followed by in-house load and stability testing. Finally, we developed a study that would test identity formation over time in a bespoke LBSN of our own creation that focused specifically on those identity processes.

than Foursquare enables the pluralization of the LBSN literature and helps minimize potential bias stemming from the use of a single application.

GeoMoments can be seen in Figure 2 and affords for the following activities based on the concept of creating a hybrid space:

- Creation of "moments" through geo-tagging of text and images-with the ability to delete or edit them;

- Creation of a user profile with a picture and other personal information;

- Friend list with the ability to add or remove friends;

- Comment system that enables threaded interactions with other users;

- "Mention" system that enables users to "tag" other users in moments and comments;

- Notification mechanism that informs the users of comments, mentions, tags, and updates to moments from friends;

- Archive of all moments posted, interactions, GPS traces, and so forth (please note that the user can only see his/her archived information, which differs from other LBSNs).

GeoMoments is an Android application that was developed by two programmers using the Android native API. We have released an open source version of GeoMoments under the GNU General Licence V3.0. The open source version of GeoMoments includes both the back-end and front-end elements, is well documented, is modularized, has a lot of locative and social functions implemented, and works in pretty much all versions of Android right out of the box. It can be requested from the authors or found at the following link: https://bitbucket.org/Geomoments/.

The design process for GeoMoments focused on creating a technological probe and not a commercial product. Or to put it another way, we wanted to evaluate bundles of affordances to amplify LBSN user performativity. The result of this probe development was as such that we did not take into consideration usage scenarios outside the scope of the main functionality of GeoMoments. To wit, as GeoMoments does not requires high-granularity GPS tracking to function as expected, we did not test or optimize for high data loads, high movement speeds of users, or GPS tracking complications caused by buildings or other environmental disturbances. 

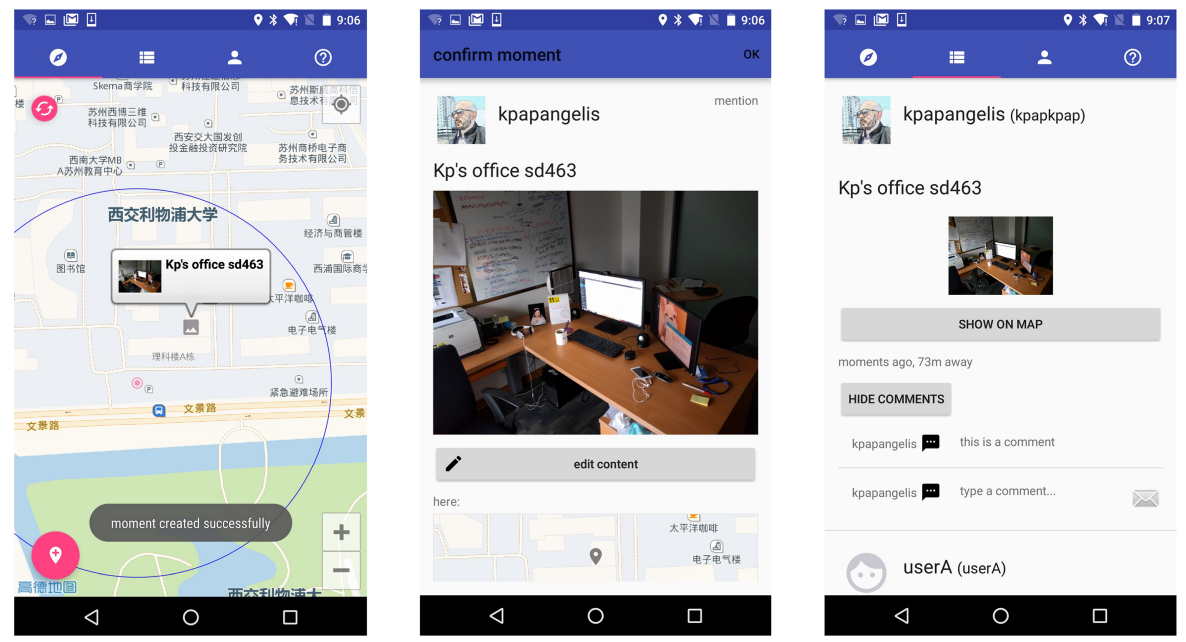

Kp's office $s d 463$

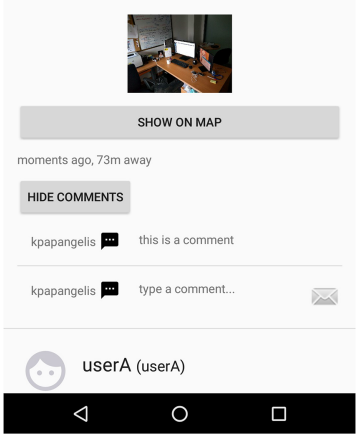

Fig. 2. The GeoMoments app interface allows users to add "moments" and interact with other users. The design of the app draws from popular social networks to lower the app's learning curve.

The desire to understand the performance aspects of users in the design of LBSNs, we based our design on the affordances of other LBSNs. For example, the affordances of GeoMoments are analogous to those of Foursquare, but also we draw from design trends and concepts already popular in other SNSs in China, such as Weixin ${ }^{5}$ and Sina Weibo. ${ }^{6}$ We did this because the behavior of the users of LBSN is believed to be shaped by the the social affordances it provides [43]. That is not to say that different affordances in different LBSNs will result in completely different behaviors, but rather that these affordances play an active role in "heightening" or "quietening" certain behaviors. To achieve the goals set forth in this study, we designed the mechanics and affordances of GeoMoments to "amplify" the playful antagonism and territoriality expressed by the participants as these would additionally amplify performativity.

This may have caused some findings to be more prominent than others. For example, the design changes that were aimed at encouraging "playful antagonistic behavior" and "territoriality" may have elicited stronger expressions of territoriality than we would have otherwise observed if we had implemented different design affordances. Similarly, these changes may have "quietened" the expression of other behaviors, such as collaboration. Modifying the design to elicit a specific behavior to study aligns with the relevant literature on the topic and is a common practice when one explores multidimensional phenomena, such as performativity and self-identity $[3,7,71]$. The present research should not be interpreted in a vacuum in relation to the design of GeoMoments, but rather in parallel with existing discourses, literature, and understandings of LBSNs more generally.

As we designed GeoMoments, we carried out a series of extensive tests in a controlled laboratory environment to test its viability and stability. Each component of GeoMoments was tested individually and with respect to other components. After testing GeoMoments in the laboratory, we recruited 20 individuals to test the performance and stability of our LBSN in a multi-user scenario, using different devices over a period of 4 weeks. Following the testing phase, we conducted extensive discussions with the participants in an informal setting and critically.

\footnotetext{
${ }^{5}$ https://weixin.qq.com.

${ }^{6} \mathrm{https}$ ///www.weibo.com.
} 
Once this initial design or testing phase was complete, GeoMoments allowed us to enable interactions both in the wild and in specific locations. Put differently, the user experience depended not only on the events on the screen but also on the players' position in the physical world. The interface was familiar in terms of look and feel, and as such our users could transfer their knowledge of other social networking platforms. This enabled a space of actions that encompasses not only what the participants can be involved in but also what they act on as they "do the city" in their everyday lives. We believe our product allowed us to amplify the aspects of LBSNs that center on performativity to examine it in depth. Figure 2 provides screenshots of GeoMoments.

\subsection{Study Design}

As noted, we sought to understand how the self, specifically the performance of the self, was realized through our application. As we had created GeoMoments using popular affordances from other products, we felt that we had created a tool that could help us realize that goal.

3.2.1 Deployment. Once the testing phase was over, we began a study involving a total of 42 students (20 men and 22 women, with an average age of 27 years) was conducted over the course of 6 weeks in the Suzhou Industrial Park (SIP) ${ }^{7}$ in the province of Jiangsu, China. The participants were recruited through flyers we handed out at the campus of the Xi'an Jiaotong-Liverpool University (XJTLU). Each participant was offered 150 CNY (approx. 22 USD) in Taobao vouchers to participate in the study. As soon as individuals expressed interest, we conducted a short prescreening discussion with them to ensure the following:

- That their phone met the technical requirements of GeoMoments (e.g., correct version of Android, over 2 GB of phone memory);

- That they were enrolled into a mobile plan with adequate data;

- That they understood what was required from them and the terms of the study.

During pre-screening interviews, we also asked if they know anyone else who was participating in the study and if they know anyone from the research team to ensure that participants did not know each other or the researchers involved with the study. Once we had 60 expressions of interests, we formed a candidate cohort that included participants who answered "no" to both questions. After cross referencing the collected pre-screen information, we formed the final cohort of 42 . All 42 participants were students of XJTLU who lived near each other and spent a substantial amount of their day at the SIP area near the campus.

\subsection{Data Collection}

The participants interacted with GeoMoments 15 to 20 times a day during the study period. The majority of interactions happened between 9 am and $6 \mathrm{pm}$. We had no inactive participants or dropouts in our study. On average throughout the study the participants spent 16 minutes every day on GeoMoments.

In total, the participants:

- Created 380 pictorial posts;

- Created 202 textual posts; and

- Interacted with each other 1,876 times, making

\footnotetext{
${ }^{7}$ Even though SIP is the area in which we conducted the study, it is not an industrial park. It is a high-density residential/office zoning district with a population of 2,000,000 that includes a mix of multifamily residential buildings, office space, educational establishments, several small shopping districts, and seven malls. You can find more information about SIP here: http://www.sipac.gov.cn/english/.
} 
Table 1. User Engagement and Data

Collection Frequencies by Week

\begin{tabular}{|r|c|c|}
\hline & Frequency & \% of Sum \\
\hline Week 1 & 178 & $7.2 \%$ \\
\hline Week 2 & 369 & $15 \%$ \\
\hline Week 3 & 371 & $15 \%$ \\
\hline Week 4 & 498 & $20.2 \%$ \\
\hline Week 5 & 491 & $19.9 \%$ \\
\hline Week 6 & 578 & $23.5 \%$ \\
\hline Total & 2,458 & \\
\hline
\end{tabular}

- 656 comments,

- 309 likes,

- 310 mentions, and

- 601 profile visits.

This made a total of 2,458 points of data collected. For a breakdown of these values by week, please refer to Table 1.

The frequency of posting and overall usage was fairly even among all the users. We believe that this was because of two reasons: (1) it was explicitly mentioned to them that they have to actively use the app everyday for the duration of the study, and (2) and they were getting notifications in their phone from the GeoMoments app every time a new post happened near them or if someone else interacted (e.g., liked) with one of the posts they made, and that "probed" them to use the app.

In detail, the frequency of usage each week is as follows. During week $1,178 / 2,458$ or $7.2 \%$ of the data was collected. In subsequent weeks, the data either remained close to the same with week 2 representing $369 / 2,458$ or $15 \%$ of the data, and $371 / 2,458$ in week 3 or $15 \%$ again. In week 4 , use increased slightly to $498 / 2,458$ or $20.2 \%$ of our total data. In week $5,491 / 2,458$ or $19.9 \%$ of our data was collected, and by week $6,578 / 2,458$ or $23.5 \%$ of our data appeared. Such a distribution of interactions is normal and on par with similar studies that involve deployment of technologies in the wild $[14,42]$.

To delve deeper into the topics relating to the goals of our study, we interviewed participants using a semi-structured approach during check-ins about the study. Each interview lasted roughly 45 minutes and included questions from a predefined set of topics with additional data made available for probing questions. The interview topics were based on topics relevant to the literature explored. These were as follows:

- The persona(s) they projected to other participants;

- Impression management;

- Issues around self-presentation and identity;

- Serendipitous, ad hoc, and playful interactions with nearby strangers;

- Reflection and memory;

- Social norms and public places;

- Authenticity;

- Expressions of territoriality;

- Attitudes toward spaces, places, and time.

Because we had the content that our participants shared during their use with the app, we were able to use it as probes during interviews. For example, the usage statistics or frequency of interactions allowed us to ask about what prompted them to use the app when they did. Further, more 
unique data such as pictorial or textual moments and interactions with others through likes and comments provided us with ways to probe more deeply than we could have when using proprietary data from apps we did not create. This approach aimed to evoke important moments, people, places, things, and events, and clarify vague or ambiguous answers or when we wanted more specific or in-depth information about a particular situation [48].

\subsection{Data Analysis}

After all data collection was complete, we transcribed the interviews and began to analyze the data. Since much of the data we were interested in came from the interviews, we spent most of our time with those data. Each interview was analyzed through thematic analysis [29].

To relate our data to both spatial and temporal dimensions literature, we did the following. First, we used the "spatial self" and as such defined "moments" as a device of self-presentation and account activity [62]. Second, we conceptualized the locations marked by user moments in a chronologically recursive fashion to reinforce the spatial and temporal dimensions that contribute to the reflective narrative and performances of a person's self-identity [27]. Third, we acknowledged GeoMoments as durational objects of "mediated memory" and treated user-generated moments as interconnected points of reference that can shape the present by bringing forward past events and project into the future $[22,60]$.

Further, as identity is multifaceted and includes both "external" and "internal" components, we used Goffman's theatrical metaphor of "front stage" and "back stage" metaphors, and we primarily considered in our analysis the externalization (manifestations of self-identity) of individuals through LBSN rather than things individuals do not externalize and require more probing (e.g., thought processes and internal schemas associated with the externalizations). ${ }^{8}$

The analysis of the data involved the following key stages: (1) familiarization of the data, (2) developing a thematic framework, and (3) coding of the data. In the familiarization phase, we transcribed all interview data. This was followed by the creation of an initial thematic framework based on a priori issues as described in the related literature, and from topics that surfaced during the familiarization phase. During the coding stage, four coders used exact quotes from the participants to inductively identify potential themes and patterns within the data and collated all the relevant coded data extracts within the identified themes.

Once the coding phase was concluded, we further refined our initial thematic framework. This was done by (1) reflecting on our process and discussing individual constructs to identify and resolve issues (e.g., clarifications of broad coding definitions), and (2) collectively reviewing the coded data extracts, and revisiting the whole dataset. This process was undertaken to not only iron-out issues but also to ascertain whether the themes "worked" in relation to the dataset, and whether any additional data within themes had been missed in earlier coding stages.

To examine the inter-coder agreement of the four coders, we used the Cohen's $\kappa$ index paired with the $\kappa$ coefficient across the coder pairs, and average $\mathrm{P}(\mathrm{e})$ values were calculated as well [1]. The agreement between the coders is shown through a $\kappa(\mathrm{m})$ of 0.788 (with $95 \%$ confidence intervals of 0.104 to 0.232 ) and $\mathrm{p}<0.0005$. Thus, we can say that their agreement was good. According to Landis and Koch [45], $0.7<\kappa<0.8$ represents a good strength of agreement, and the confidence interval indicates that the coding is reliable, non-random, and in line with previously published inter-coder

\footnotetext{
${ }^{8}$ It should be mentioned that in our analysis, we only focused on the self-identity of individuals and how it unfolds as time goes by and did not look into other self-identity structures (e.g., group, social). As such, and in coming in line with the literature and our interpretative framework, we considered all (1) externalized interactions through GeoMoments (e.g., history of activity, narrative through pictorial and textual posts) and (2) activities that aim in re-shaping one's front stage activity (e.g., editing posts, deleting posts, liking other people's posts) as front stage actions as they were designed to be seen by others and to improve one's public self-image and hence included them in our study.
} 
reliability estimates obtained from coding similar constructs. The marginal distributions of coding also did not indicate prevalence or bias problems. This suggests that Cohen's $\kappa$ was an appropriate index of inter-coder analysis [25].

Once we were confident that our thematic framework fitted our dataset and we were able to tell a coherent story, we concluded the analysis process.

\section{FINDINGS}

As noted, we designed GeoMoments to foreground sociality, performativity, identity formation, and identity maintenance. Many of the following findings are perhaps more directly observable in GeoMoments than those in LBSNs where some of these behaviors may be partially obfuscated by other aspects of the product's user experience. Despite that purposeful foregrounding, the present research provides much-needed experiential data of users engaged with a product that was specifically designed to heighten their temporal identity. Each of the following sections is divided into weeks to show directly what we intended to show.

\subsection{Weeks 1 and 2}

4.1.1 No Posts on a "Blank Canvas". During the first few days of the deployment, only a handful of pictorial moments were posted and even fewer textual moments were recorded. As time went by, however, participants started posting more content throughout the city. These postings were mainly done in clusters. When we asked our participants about this behavior, many of them mentioned that that they were feeling more comfortable sharing content in locations where other individuals had already shared content. As Mary explains:

When I first use GeoMoments I did not know what to post or where [...] I was only posting moments where other have posted before [...] It felt more comfortably and appropriate to do that. (Mary)

4.1.2 Posting Behavior Mimicry. Our data shows that participants were not only posting moments that where geographically close to other people's posts, but that the content of their posts was also similar. For example, a participant took a selfie in front of a statue and within days there were several other participants posting similar selfies in front of the same statue. This mimicry in posting behavior resembles the phenomenon of social synchrony or "the tendency of a large group of people to perform similar actions in unison, in response to a trigger" [17]. This resonates with de Souza e Silva and Frith's understandings that LBSN can potentially foster the creation of a shared identity relating to a location [19].

4.1.3 Posting Interactions Helps Change Perceptions of the City. This behavioral mimicry identified earlier, then, caused several participants to change their perceptions about certain areas of the city. As Joe notes:

I always used to avoid Jinjihu dadao as it is very busy and dangerous, especial near Ligondi $[\ldots]$ however after seeing a lot of people posting around there I decided to go and check out their moments, and this is when I realized that the highway has been renovated and is not as busy as I thought it would be [...] so after seeing that I decided to change my route and go through the highway instead of going through Dongyan lu. (Joe)

This finding accordingly supports surrounding literature on locative media and the suggestion that LBSNs have the potential to motivate participants to re-negotiate perceptions of urban 
structures, disrupt locative habits, and modify approaches toward mobility [30] and physical space $[8,50]$.

\subsection{Weeks 3, 4 and 5}

4.2.1 Content Mimicry Lessens as Users Build Deeper Interactions. After 12 days, participants started posting moments more regularly. This illustrates that there was an adaptation period where participants familiarized themselves with the technological affordances of GeoMoments. Around the same time, we also observed that some participants started to actively mock content mimicry posted during the early adoption period. For example, a participant in our study posted a moment in an area with high traffic that included the Suzhou Skyline from across the Jinji Lake with the Gate of Orient in the center. The Gate of Orient is a landmark building of the skyline in the city center of Suzhou and is jokingly known as "pants building" due to its shape. Other participants then played upon the pants building joke by taking photos of their pants. This escalated quickly, and after a few days, some participants started posting selfies with pants in the background clearly mocking the other posters. As Ed explains:

I found it very funny that everyone was copying each other and posting photos of the same things in the same places. So, I decided to have some fun. I never thought that other would think that what I done is cool [...] and would do the same. (Ed)

It should be noted that some of the individuals participating in this activity were the same people who posted the initial photos of the skyline. Andy further expands:

At the beginning they were mocking my moment! I got really frustrated but I kept cool and made fun of myself by posting an over exaggerated photo to show to everyone that I'm chill and don't take things serious. (Andy)

4.2.2 Participants Became Aware of Others and Start Curating Their LBSN Digital Identity. Following the incident noted previously, our data found that some participants consequently became more aware of what they posted in GeoMoments. Ann expands upon this point:

I am very careful what to post online. I know that John is using GeoMoments and last thing in the world I want is for him to see my photo or someone else to show him my photo. I am not going to put myself in the bin like that. (Ann)

For Ann, then, the posts in GeoMoments are a way of continuing her identity narrative as it "shows who you are" to the other participants. This was echoed by several participants and mirrors the findings of other studies that have made similar observations [15, 35]. Accordingly, it is apparent that GeoMoments allows participants' identities to be forged through the sharing of a place and its perceived connotations through pictorial and textual moments. An important element of this process is the awareness that moments can conceivably be seen by others, or as Saker [58] refers to it, "the affirmation of identity through the potential of surveillance." Tony further expands on this:

I was going through my Moments before this interview. I realized that most of my moments were in the SIP area when I was out at night $[\ldots]$ and even though I spent almost 5 hours every day in the library of XJTLU this semester I don't have any moment there [...] I made this mistake once and I was called a geek by Josh and Helen [...] no way in hell I am doing this again [...]. Another place I am going often and have no moment is the noodle store in Hanlil center. I am sure that if I post a moment there people will call me cheapskate [laughs]. (Tony) 
This type of reply was very common among our participants. Many participants did not like being seen in certain places. This was due to the influence those places would have on the self narrative that they were crafting in GeoMoments.

4.2.3 Users Post in Areas That They Have Ties with or That They Want to Claim. When we asked participants about where (or where not) they were posting moments, it turned out that they posted moments mainly in areas they had parochial ties with, or areas that they considered of high value to others (e.g., malls) to "claim areas" as their own to make sure "everyone knew that they were locals in this area," and avoided posting moments in or claiming areas that would make them "seem uncool." As the following extract attests:

I chose to post a lot of moments around the area near my house and Linkcity mall. I posted a lot of interesting things around the area my house is as I want to show to others that I am local here. [...] To be honest I don't want others coming in posting their food or pets. [...] but I posted a lot around the linkcity and other malls [...] to look cool to others. I mean if you post a lot in an area others see you as local. Being local at the mall is big deal. [...] you show to others that you are regular at a cool place. (Jess)

This supports the suggestion that some participants understand their connection to certain places GeoMoments as being representative of a certain lifestyle, and that the moments the participants post were seen as part of an ongoing narrative of the self.

The terms local and claim were used extensively by participants when discussing different areas. It should be noted that GeoMoments does not have a way for participants to articulate identity claims over places. As a result, the participants "claimed" areas by posting a high number of moments inside them. The content of these moments varied and included, among others, informative posts (e.g., the bus stop has moved), selfies, and posts mocking other participants.

4.2.4 Status and Post Usefulness Matter When Claiming an Area. When someone threatened a "claimed" area, one of the principles that prompted a defense of that area is whether the claimer was perceived as having a legitimate claim on the place. As Jesse states:

I used to have the most moments around the Linkcity mall [...] in restaurants, Starbucks etc. Everyone knew I was local. Now Korra came here and started posting moments. She didn't have a single post before. Now she has 12 and I have 21. She thinks that she is better than me. (Jesse)

Such behavior was evident among most participants when someone challenged their local status by posting moments in their "claimed" area, with the degree of concern seemingly increasing the more participants understood themselves as having a connection to the environment prior to this LBSN. In some cases, this led participants to take extreme measures. For example, one participant posted a selfie holding a piece of paper and claiming an area and declaring it a "no moments zone" for anyone else except himself.

Places that were highly contested were often places where the others' claims were seen as illegitimate or were perceived by the majority of participants as being of high value to "own" in the eyes of other participants. For example, a participant described how another participant "came and stole my post and my area [...] I wrote about the bus stop not working. Why has he started posting his selfies here? Obviously, I post important things and he does not."

Several participants mentioned similar things especially with regard to moments containing locational information that was deemed useful. This illustrates that a subset of participants perceived hierarchy over an area not only based on the parochial ties with that area, or the perceived value of 
the area to others, but also on the usefulness of the information one provided through their claims on an area. It should be noted that 16 out of 42 participants used the word "steal" when talking about other participants "taking" their area from them. This speaks to the perceived legitimacy of someone's claim to a particular place, that it confers value worthy of theft, and suggests the stealing of a hierarchical position from which one can make a claim over a place. This resonates with Guha and Birnhotlz's [35] proposal that the visibility of check-ins in Foursquare can lead to tension.

4.2.5 Participants Create Social Connections Through "Digital Self” Similarities with Other Users. In addition to this, during our study we observed various social interactions between participants, instigated by the narrative they chose to perpetuate through GeoMoments. These are worth reporting upon especially if we consider that previous research has illustrated that in large cities people tend to create distance from each other, despite the conditions of closed proximity [6]. This was particularly prevalent in participants who had similar self-identities, like Helen who is a "known foodie" and used GeoMoments "as a way to document the location of street food vendors in Suzhou." For Helen, GeoMoments is not only a way to capture and showcase street food but also a way to connect with other participants who have similar interests, such as Rob who used GeoMoments as "a journal to document interesting things" he came across, or Alex who also defined himself as a "foodie" and used GeoMoments to posts pictures of food from various restaurants he visited and to comment on other participants' moments relating to food. As Helen explains:

I started posting street food vendors in Suzhou and soon others joined in commenting about their food quality, other places they have seen them etc [...] then I noticed that there were others that were posting food related topics and I contacted them [...] they responded and we started a foodies group where not only meet in real life but we try to keep track of the location of street food vendors, review restaurants etc. (Helen)

Alongside forging and perpetuating identity-based narratives, GeoMoments also provided participants with the opportunity to connect with other individuals who shared similar self-identities. This has the potential to affect their self-identity in LBSN:

I was using GeoMoments to capture the tasty food around Suzhou [...] a few people contacted me if I would be interested to join their hunt for tasty food around Suzhou. I thought that it was cool and I agreed. Now a few weeks later I am hunting down street food vendors and going to all these tiny restaurants with them [...] people make fun of me in my comments and call me the "food review guy." I don't take offence. It is very funny as all of my moments are about food! People must think that I am crazy about street food [laughs]. (Alex)

The methods that Helen and Alex use to describe themselves in relation to their usage of GeoMoments fold into comparable narratives illustrating that GeoMoments provided new social opportunities, which fed back into their ongoing personal narratives.

4.2.6 Participants Continuously Curate Their LBSN Identity. Our data explicitly demonstrates that the sharing of location through moments not only has significant ramifications with regard to the identity practices of participants but also to the construction of meanings associated with a space $[9,15,35,46,65]$. GeoMoments allows identity to be "an on-going project that is constantly being modified, updated and safeguarded" and "constructed through certain techniques or practices" through "the multiplicity of ways in which individuals constitute their identities in a creative and constructive fashion" [64]. As such, by sharing location-based moments, GeoMoments contributes to the creation of identity, influencing the way that it is revised, 
perpetuated, and safeguarded. Identity becomes something that is curated. As Saker posits, "the mode of construction (of identities) is significant here, as in this instance concomitant identities are in part shaped through location, and an awareness of what location might mean. This is established by the fact some participants subsequently felt compelled to share their location at a particular place" [59].

This was echoed throughout our study by almost all of the participants. As Jonathan explains:

I would only leave moments in cool places. I would never leave a moment at university, work or in the shabby and rundown parts of the city. If I do that than other may think that I hang around and I'm local there [...] [laughs] I try to maintain a cool persona [laughs] I have even asked people to remove my mentions from moments in uncool places [...] my image my image [in a squiggly voice, laughs] [...] the only time that I had ever left a moment at the place I would not normally leave moments is when I passed my exams. I wanted everyone to know that I was a senior now. (Jonathan)

In this passage, Jonathan wants his moments to fit within a predefined narrative that conforms with the "cool" identity he wants to project through GeoMoments. He only broke from that narrative to use GeoMoments to make explicit his new identity of "senior student." This demonstrates that Jonathan wants his performance through GeoMoments to be seen by others in order for their performed identities to be realized. This is supported by Saker's observations that "by becoming more aware of the importance of location to certain friends, [a user] consequently becomes aware of what these locations might mean in terms of [his/her] identity" [27], as well as other studies that have examined Foursquare and impression management [15, 35, 62].

\subsection{Week 6}

4.3.1 Constructed Spatial Self-Identities as a Means for Reflection for Certain Participants. The construction of ongoing narratives, such as Jonathan's, relies on the participants being aware of his or her interactional history with the system, places, and with other participants. GeoMoments enables the participant to easily log and recall this history of actions. Some participants used this function as a historical locational diary, which they used to curate their narratives. As Tanya explains:

I like that GeoMoments keeps a history of all my moments, comments and mentions $[\ldots]$ I visit it very often [...] it is like keeping a trail of the history of my life online. (Tanya)

Similarly, Bob used GeoMoments as a locational historical diary and also as a tool for reflection:

I used [GeoMoments] to keep track of where I've been and all the cool things I have seen [...] but I also [through using GeoMoments] started noticing some things. That for example I have over 30 moments of me drinking in Ligongdi and only 3 moments at or nearby the gym [...] this is not good [...] once I noticed that I started being more proactive and I am now trying to go to the gym more often. (Bob)

The desire to keep a historical log of moments is indicative of a desire to keep track of an individual's narrative through GeoMoments. This can aid in curating a self-identity through LBSN, such as Mary's "shop-a-holic" self. At the same time, it can also make the individuals more aware of their actions, and as such provide nudges for reflection and integration into self-narratives. These nudges (or micro-nudges) have the potential to significantly alter the self-identity and performance narrative of an individual, as there is evidence that he or she can facilitate reflection and behavior change. For the most part, this is the case for Mary; by reflecting upon her shop-a-holic 
self portrayed through GeoMoments, she decided to alter her "self" by announcing her new self to her perceived audience-the other participants of the system.

For participants such as Tanya, Mary, and Bob, then, GeoMoments allows them to record and replay their location history. This acknowledges GeoMoments as akin to Chun's [13] "digital data banks" that provide a "possibility of knowing being, by being able to project oneself from the past into future action." In addition, this can be seen as an inorganic organization of memories that are being reflected upon and allow the forging, revision, perpetuation, and safeguarding of data about oneself.

Furthermore, in our study, some participants used GeoMoments to document spatial movements and the location of places. This was particularly important to a subset of participants who were new to Suzhou and unfamiliar with the city. Boris said this:

I just moved to Suzhou. I am not familiar with the city at all. [...] I used GeoMoments as way to record where the supermarket is, which bus stop to take, and where to go buy water and stuff $[\ldots]$. (Boris)

For Boris, GeoMoments was used to familiarize himself with the city of Suzhou. This enabled Boris to structure the present by focusing on the future, as well as to structure the present by bringing forth the past. This illustrates that the timeline is in many respects determined by the user and is not "a fixed manipulation of temporality through quantification of time" [60].

4.3.2 Participants Curate or Recreate Their Spatial Future Self Based on Archived Location Information. As mentioned earlier, GeoMoments was perceived not only as a device to record and replay location history but also as a way to guard a certain image that would otherwise wane in time, therefore echoing the findings of Saker and Evans [59] regarding the "accumulative self" in the context of Foursquare. This transcends the (oftentimes) ephemeral nature of social networking, as it enables the individual to acknowledge certain aspects of spatial pasts and act upon them in the present in a way that supports the maintenance of the current identity the user wants to portray. This became apparent when participants such as Bob discussed how they would browse old moments to "see what they were posting back then" so as to identify and use certain elements of their past spatial self to re-evaluate their present spatial self. For Bob, the aspects he wants to maintain are things that conform with his current perception of "coolness," and the aspects he wants to weed out is behavior and posts that make him feel "like a fool" in the eyes of others. As Bob details:

When I first started using GeoMoments I was posting a lot of dumb stuff [...] making fun of others and such [...] but I also [realize looking at my history that I] posted some quite cool things [...]. [Nowadays] I only post cool things for others to see when they are where I used to be. [...] I want them to see my posts and say that I am doing interesting things and I am cool [...] and to be honest it is true because I AM [emphasis] really cool [laughs] and yeh [laughs] [...] as such I stopped posting dumb thing [laughs]. (Bob).

Similarly, instead of looking through their history and deciding to maintain certain elements of their past self in representation of their present self, other participants decided to delete or hide the old moments that did not conform with the present identity they portray. As Jesse states:

I checked my [post] history recently. I posted a lot of things that I didn't like. [pause to think] That don't show who I am. Don't reflect me. [pause to think] So, I just deleted them. No point having them there. [....] others will see them on my history and this will reflect bad on me. (Jesse) 
This curation of present self-identity by taking into account or (in some cases) completely deleting past expressions of self is potentially indicative of an attempt to guard present-day self-identity based on past expressions of self. As such, it becomes clear that identity in GeoMoments is temporal and created and recreated through the recording and replaying of space and location. This ongoing negotiation illustrates well-defined links between possible modifications of the past self through the present self, potentially affecting the future self. This results in the expressions of self in LBSN being chronologically recursive, with the modification of historical moments effectively altering the current self.

Moving forward, then, our data suggests that LBSNs that enable participants to delete or hide historical location activity not only act as "mediated memory objects" but also as nebulous memory containers where the past and the present bleed into each other through re-conceptualization or modification of the past to reflect the present while "anticipating the future and drawing on the past to structure the present" [26]. The desire for self-identity homogeny between the past and present that participants portrayed in our study illustrates that individuals perceive the expressions of self not only as "a thing of now that ignores the past" but also as a trajectory of past archived "experiences" all culminating and loosely aligning to the present self. At the same time, the archiving functionality of GeoMoments is important here, as unlike Foursquare, accumulative archives cannot be seen by other users. In other words, and significantly, this demonstrates that our participants were not simply focused on this aspect of the spatial self because of its desired meaning for other users but, more impactfully, what it validated in themselves.

\section{DISCUSSION}

Within GeoMoments, the temporality of identity is created and maintained in similar ways as any software with affordances that are social in nature. Upon opening GeoMoments for the first time, users learn how to use it. This is akin to establishing a front stage based on the negotiation between affordance, back stage identity, and knowledge of the platform. Once that front stage is established, it becomes a means through which the platform and user communicate to others on that platform. This process was observed in our users. Accordingly, we will now discuss how this process was established.

As noted, in the first week of our study, participants primarily posted content where prior content existed and their posts imitated this earlier content. GeoMoments fostered a shared creation of identity among users through that mimicry. It also encouraged playful interactions through comments and posts some of which resulted in the creation of a communal identity vis-à-vis a location (neighborhood) in the city. This communal identity led to several participants reconsidering their perceptions about those areas of the city.

As evidenced in our study and the literature, this process has the potential to overwrite the existing meaning of places, and to facilitate social situations where participants can appropriate space, enabling them to reshape its meaning, history, and significance $[12,49]$. Therefore, the interactions afforded through GeoMoments can be used as a way to allow participants to experience familiar surroundings (or surroundings that the user might have strong preconceptions about) in different ways. Just as Saker [27] observed, for some users the environmental information Foursquare provided allowed those to feel local in an unfamiliar environment. In the context our study, GeoMoments had the power to transform known spaces into environments that were suddenly open to new discoveries.

Put differently, this LBSN was effectively able to "make the familiar strange" [2]. This bridges the notion that our practices in the urban setting are statements open to interpretation and thus establish the power structures of the city. GeoMoments serves as that bridge in that it can be used in conjunction with other interventions aimed at altering perceptions and can challenge 
preconceived notions regarding the urban landscape. LBSNs in such scenarios act as an interface that renders practices and interactions between participants, space, and place readable. As a result, this may overwrite (or potentially reinforce) embedded power structures, disrupt habits, and make the familiar strange and negotiable.

During the end of week 2 but mainly in weeks 3 and 4, the mimicry of existing posts lessened and participants engaged with GeoMoments in more diverse and varied ways. This variance in the middle of the study was when our users were curating and starting to safeguard their digital identities. Identity curation in GeoMoments was achieved through how GeoMoments archived their locational moments. In essence, where they went and noted on GeoMoments was the literal, temporal, and actual expression of their identity as a person. At certain points, this created uncomfortable reflections for our participants by reminding them, for instance, how much they had been drinking and how little they had worked out. Our findings echo the literature focusing on the performative side of marking one's location through LBSN in the context of impression management $[27,62]$, and self-representation and identity $[4,24,63]$.

For many of our participants, the ability to accumulate locative traces that were outwardly congruent with their inward sense of self. This congruence allowed our participants to perform an expression of self that felt less performative and more authentic as its temporality increased. To wit, the longer their dominance in a space went on, the more authentic that space became as part of their identity. The mediation of self through LBSN therefore adds an extra layer of veracity to the identities performed resulting in participants experiencing their locative identities as articulations of the real self. These articulations are predicated on the digital representations of physical activities performed through GeoMoments. This has been observed in previous studies to a limited extent and has been theorized as happening in order that the "identities feel more consistent by aligning front stage and back stage behaviors" [59].

It is our belief that this can also be attributed to the interplay of the polysemic nature of place in relation to preconceived notions that individuals have about a particular location and its inhabitants that, for the purposes of GeoMoments, was further mediated by time spent at location or tenure. It is also our suggestion that this interplay was responsible for some of the adversarial conduct we observed, selves attempting to manifest at the same locations in GeoMoments. This antagonism mainly manifested as territorial claims of areas in GeoMoments with textual and pictorial moments. When their "claimed" areas were challenged, the participants exhibited antagonistic behaviors that included posting more moments in their area to legitimize and communicate their claim, or even making posts directly communicating that they had claimed an area. All of the participants in our study tried to claim and "protect" spaces where they lived, their "home territory," even though the home territory is not a technical affordance featured in GeoMoments.

This wording and protective behavior indicates territoriality and is indicative of an attempt to guard self-identity through the mechanics of LBSN. In many ways, we would suggest this mediated performance offers more reach and authority in making territoriality obvious and accountable than a single person could through everyday physical practice in a space abstracted from locative media. By making virtual claims over a particular geographic area, users do not have to actually exert control over others in a physical sense but by virtually taking over one's connection to a place, which represents power within that area. Encroaching on that power not only threatened identity but also provided enemies, provocateurs, and outsiders with names, faces, and spaces. Loss of those areas represented a certain loss of self, whereas gaining dominance in an area highlighted a shift in power that had ramifications for those around that area that often took the form of notes and moments.

As the study entered its final stretch in late week 4 and throughout week 5, some participants engaged with spaces and places in a way that was shaped by the collective identity and social 
interactions of individuals they communicated with through GeoMoments. For example, whereas some participants viewed one neighborhood one way communally, this was challenged by other participants who began to encroach on that communal identity. This was the result of a "heightened cognizance of the nexus between place, identity, and surveillance" [59]. Through our interviews, it became clear that the sharing of moments and geo-traces of activity not only had significant ramifications with regard to the identity of individuals but also to the constructed meanings and perceptions associated with space and place. This behavior can be attributed to the consideration of who might be a potential audience for the user's moments, and to the uncertainty of who is part of this potential audience. As such, it is evident by our study that the way individuals share locational information that contains locative connotations tends to conform to the front stage performance of the "self." And this results in the ways that the meaning and perception of space changes resulting in certain places becoming more desirable to add entries about than others.

In the last week of the study, the participants who were more deeply engaged with GeoMoments relied on their history of interactions with the system and other participants to maintain their identity narrative. The desire to keep a historical log of moments is indicative of a desire to keep track of an individual's narrative, the individual's journey through space, as captured by GeoMoments. By viewing the log, users are able to curate a self-identity, make one more aware of their actions, and as such provide nudges for reflection and integration into self-narratives. We suggest that the reflection we observed in our study is facilitated primarily through nudges that occur during the use of LBSN as a locational historical diary and the perceived connotations that these actions have in the potential audience of these.

These nudges have the potential to significantly alter the self-identity and performance narrative of an individual including moments of being antagonized by outsiders or aggressed by provocateurs (literally a record of war, in those cases). This is further illustrated by individuals deleting past moments that do not align with current expressions of self (or be highlighted when victorious), as there is evidence that they can facilitate reflection and therefore contribute to behavior change. Following this, we believe that LBSN usage, as a "mediated memory object" may support awareness building in temporal terms. This additionally has normative influence on the participants as the locational past permeates into the present and potentially affects their future behaviors. The discussion of temporality, the journal, learning how to use GeoMoments, and how these things changed throughout the study highlight the relevant (and rather limited) literature that the locational past has strong potential to shape the present and future expressions of self. As a result, temporality could potentially (and actually in the case of our participants) affect user behavior and self-expression (e.g., [26, 54, 67]). Furthermore, our study casts a meaningful light on the "spatial self" [62] as a suitable framework from comprehending LBSN usage as it pertains to self-identity. Indeed, while Schwartz and Halegoua do touch on the archival aspect of locative media, this affordance is not the focus of their discussions. As a corollary to this, what our study effectively demonstrates is the function of temporality in this context. Here, the use of LBSN does not simply change over time in response to the self-shifting outside of this application. More precisely, documented changes to the self are symptomatic of the self-documentation that LBSNs enable.

\section{CONCLUSION}

Overall, our discussion of the interplay of self-identity and temporality in LBSN illustrates that it can be used to describe various person-to-person and person-to-place interactions, as illuminated previously. For many participants, the locative functionality of LBSN allowed them to perpetuate and affirm their territorial ties, and in so doing reflectively set their perceived sense of self. In the context of identity and performances of the self, then, LBSN usage does not simply affirm 
the self through the presentation of place and the abstracted symbolism different places exhibit. For some participants, at least, what is equally important is the spatial positioning of place in the context of its surrounding environment. The decision to physically and digitally interact with a specific location suggests something about the self that is congruent with this site, just as it serves to territorialize the spatial self within a certain terrain. Consequently, the temporal and digital inscription of place does more than represent the self, thus digitally inscribed; this performance correspondingly asserts the self through the implicit suggestion that the self and the surrounding environment are intimately intertwined. Although previous studies have examined LBSN in the context of identity, the connection between performances of the self, locative media and temporality have not been as explicitly explored as they have been here. Within the canon of locative media, it is important that this connection is made to better understand the spatial implications of LBSN usage. In sum, our work contributed to the discourse of locative media and identities by adding "temporality," a dimension that connects the hybrid space and people's identity reshaping process through LBSN.

Limitations of this research mainly relate to issues regarding the nature of our study and the designed affordances of GeoMoments. For example, as mentioned earlier, one can argue that the relatively low number of participants and the rather limited area of our study may have affected the participants and as such the shared vernacular, etiquette, culture, and expressions of "self" in relation to territoriality can be partially attributed to "small network dynamics," "the group norming effect," or to strong "weak ties" [68]. Similarly, it can be argued that the designed affordances of GeoMoments may have affected the participants and might have caused some findings to be more prominent than others-for example, the ease of access to the historical geo-traces of activity of a user may have elicited stronger expressions of reflection than we might have otherwise observed. As a resell, the findings that emerged from exploring this study should be treated as exploratory insights that supply a starting point for understanding self-identity narratives through locative performances in the context of durational LBSN usage. We therefore suggest that future research should (1) explore the interplay of LBSN and everyday life of the participants in a broader context utilizing alternative methodologies, study designs, and approaches under different scenarios; (2) investigate of temporal components of a system and the relationship between temporality and identities in locative media; (3) and explore whether temporality is a mediator between the hybrid space and the way people reshape their identity. Likewise, it is also our suggestion that these studies should engage with LBSN usage and performances of the self as it pertains to longer temporalities. These studies should not be explored in a vacuum but rather be linked to and explored in relation to relevant theories (e.g., technology adoption theories [47]).

Further, it is our belief that since our study sheds light on the interplay of identity and temporality, it can potentially be used as a stepping stone for human-computer interaction and communications researchers to design temporal components of a system. However, further work is required for this. For example, further work is needed to explore whether early application usage functions as a form of social learning where users take their performative cues from other users and so on. It is our contention that by doing so, we will get a better understanding of performative use of LBSN as they are accumulatively embedded in the everyday life of users.

\section{REFERENCES}

[1] Mikel Aickin. 1990. Maximum likelihood estimation of agreement in the constant predictive probability model, and its relation to Cohen's kappa. Biometrics 46, 2 (1990), 293-302.

[2] Mona Baker and Bolette Blaagaard (Eds.). 2016. Citizen Media and Public Spaces: Diverse Expressions of Citizenship and Dissent. Routledge, London, UK.

[3] Jeremy Birnholtz, Colin Fitzpatrick, Mark Handel, and Jed R. Brubaker. 2014. Identity, identification and identifiability: The language of self-presentation on a location-based mobile dating app. In Proceedings of the 16th International 
Conference on Human-Computer Interaction with Mobile Devices and Services (MobileHCI'14). ACM, New York, NY, 3-12. https://doi.org/10.1145/2628363.2628406

[4] Danah M. Boyd and Nicole B. Ellison. 2007. Social network sites: Definition, history, and scholarship. Journal of Computer-Mediated Communication 13, 1 (Oct. 2007), 210-230. https://doi.org/10.1111/j.1083-6101.2007.00393.x

[5] Liam Bullingham and Ana C. Vasconcelos. 2013. 'The presentation of self in the online world': Goffman and the study of online identities. Fournal of Information Science 39, 1 (Jan. 2013), 101-112. https://doi.org/10.1177/0165551512470051

[6] Judith Butler. 1988. Performative acts and gender constitution: An essay in phenomenology and feminist theory. Theatre fournal 40, 4 (Dec. 1988), 519. https://doi.org/10.2307/3207893

[7] Susanne Bødker. 2015. Third-wave HCI, 10 years later-Participation and sharing. Interactions 22, 5 (Aug. 2015), 24-31. https://doi.org/10.1145/2804405

[8] Scott W. Campbell and Nojin Kwak. 2011. Mobile communication and civil society: Linking patterns and places of use to engagement with others in public. Human Communication Research 37, 2 (April 2011), 207-222. https://doi.org/10. 1111/j.1468-2958.2010.01399.x

[9] Michel de Certeau. 2013. The Practice of Everyday Life (2nd ed.). University of California Press, Berkeley, CA.

[10] Alan Chamberlain, Mads Bødker, Adrian Hazzard, David McGookin, David De Roure, Pip Willcox, and Konstantinos Papangelis. 2017. Audio technology and mobile human computer interaction: From space and place, to social media, music, composition and creation. International fournal of Mobile Human Computer Interaction 9, 4 (Oct. 2017), 25-40. https://doi.org/10.4018/IJMHCI.2017100103

[11] Alan Chamberlain, Mads Bødker, and Konstantinos Papangelis. 2018. Sounding out ethnography and design: Developing metadata frameworks for designing personal heritage soundscapes. Fournal of the Audio Engineering Society 66, 6 (June 2018), 468-477. https://doi.org/10.17743/jaes.2018.0025

[12] Martin J. Chorley, Roger M. Whitaker, and Stuart M. Allen. 2015. Personality and location-based social networks. Computers in Human Behavior 46 (May 2015), 45-56. https://doi.org/10.1016/j.chb.2014.12.038

[13] Wendy Hui Kyong Chun. 2013. Programmed Visions: Software and Memory. MIT Press, Cambridge, MA.

[14] David Corsar, Caitlin Cottrill, Mark Beecroft, John D. Nelson, Konstantinos Papangelis, Peter Edwards, Nagendra Velaga, and Somayajulu Sripada. 2018. Build an app and they will come? Lessons learnt from trialling the GetThereBus app in rural communities. IET Intelligent Transport Systems 12, 3 (April 2018), 194-201. https://doi.org/10.1049/ietits.2016.0216

[15] Henriette Cramer, Mattias Rost, and Lars Erik Holmquist. 2011. Performing a check-in: Emerging practices, norms and 'conflicts' in location-sharing using Foursquare. In Proceedings of the 13th International Conference on Human Computer Interaction with Mobile Devices and Services (MobileHCI'11). ACM, New York, NY, 57-66. https://doi.org/10. 1145/2037373.2037384

[16] Kathleen M. Cumiskey and Larissa Hjorth. 2015. Mobile Media Practices, Presence and Politics: The Challenge of Being Seamlessly Mobile. Routledge.

[17] Munmun De Choudhury, Hari Sundaram, Ajita John, and Doree Duncan Seligmann. 2009. Social synchrony: Predicting mimicry of user actions in online social media. In Proceedings of the 2009 International Conference on Computational Science and Engineering. IEEE, Los Alamitos, CA, 151-158. https://doi.org/10.1109/CSE.2009.439

[18] A. de Souza e Silva. 2006. From cyber to hybrid: Mobile technologies as interfaces of hybrid spaces. Space and Culture 9, 3 (Aug. 2006), 261-278. https://doi.org/10.1177/1206331206289022

[19] Adriana de Souza e Silva and Jordan Frith. 2012. Mobile Interfaces in Public Spaces: Locational Privacy, Control, and Urban Sociability. Routledge. https://doi.org/10.4324/9780203123966

[20] Adriana de Souza e Silva and Daniel M. Sutko. 2008. Playing life and living play: How hybrid reality games reframe space, play, and the ordinary. Critical Studies in Media Communication 25, 5 (Dec. 2008), 447-465. https://doi.org/10. $1080 / 15295030802468081$

[21] J. Donath and D. Boyd. 2004. Public displays of connection. BT Technology fournal 22, 4 (Oct. 2004), 71-82. https: //doi.org/10.1023/B:BTTJ.0000047585.06264.cc

[22] Sophia Drakopoulou. 2017. "We can remember it for you": Location, memory, and commodification in social networking sites. SAGE Open 7, 3 (July 2017), 215824401771202. https://doi.org/10.1177/2158244017712026

[23] Stine Ejsing-Duun. 2016. Participatory urbanism: Making the stranger familiar and the familiar strange. In Citizen Media and Public Spaces: Diverse Expressions of Citizenship and Dissent. Critical Perspectives on Citizen Media, Vol. 1. Routledge.

[24] Nicole Ellison. 2013. Future Identities; Changing Identities in the UK: The Next 10 Years. Technical Report. The Government Office for Science, London, UK. https://www.gov.uk/government/publications/future-identities-changingidentities-in-the-uk.

[25] Barbara Di Eugenio and Michael Glass. 2004. The kappa statistic: A second look. Computational Linguistics 30, 1 (2004), 95-101. https://doi.org/10.1162/089120104773633402 
[26] Leighton Evans and Palgrave Connect (Online service). 2015. Locative Social Media: Place in the Digital Age. Palgrave Macmillan. http://www.palgraveconnect.com/doifinder/10.1057/9781137456113.

[27] L. Evans and M. Saker. 2017. Location-Based Social Media: Space, Time and Identity. Springer International Publishing. https://books.google.com.hk/books?id=j70HDgAAQBAJ.

[28] Jason Farman. 2012. Mobile Interface Theory: Embodied Space and Locative Media. Routledge, New York, NY.

[29] Jennifer Fereday and Eimear Muir-Cochrane. 2006. Demonstrating rigor using thematic analysis: A hybrid approach of inductive and deductive coding and theme development. International fournal of Qualitative Methods 5, 1 (2006), $80-92$.

[30] Jordan Frith. 2013. Turning life into a game: Foursquare, gamification, and personal mobility. Mobile Media \& Communication 1, 2 (May 2013), 248-262. https://doi.org/10.1177/2050157912474811

[31] Jordan Frith. 2014. Communicating through location: The understood meaning of the Foursquare check-in: Foursquare and mobile coordination. Journal of Computer-Mediated Communication 19, 4 (July 2014), 890-905. https://doi.org/10 $1111 /$ jcc4.12087

[32] Erving Goffman. 1990. The Presentation of Self in Everyday Life. Penguin, London, UK.

[33] Lewis Goodings, Abigail Locke, and Steven D. Brown. 2007. Social networking technology: Place and identity in mediated communities. Journal of Community \& Applied Social Psychology 17, 6 (Nov. 2007), 463-476. https://doi.org/ 10.1002/casp.939

[34] Eric Gordon and Adriana de Souza e Silva. 2011. Net Locality: Why Location Matters in a Networked World. WileyBlackwell, Chichester, West Sussex.

[35] Shion Guha and Jeremy Birnholtz. 2013. Can you see me now? Location, visibility and the management of impressions on Foursquare. In Proceedings of the 15th International Conference on Human-Computer Interaction with Mobile Devices and Services (MobileHCI'13). ACM, New York, NY, 183. https://doi.org/10.1145/2493190.2493209

[36] L. Hjorth. 2011. Mobile@game cultures: The place of urban mobile gaming. Convergence: The International fournal of Research into New Media Technologies 17, 4 (Nov. 2011), 357-371. https://doi.org/10.1177/1354856511414342

[37] Bernie Hogan. 2010. The presentation of self in the age of social media: Distinguishing performances and exhibitions online. Bulletin of Science, Technology \& Society 30, 6 (Nov. 2010), 377-386. https://doi.org/10.1177/0270467610385893

[38] Andrew Hoskins. 2011. Media, memory, metaphor: Remembering and the connective turn. Parallax 17, 4 (Nov. 2011), 19-31. https://doi.org/10.1080/13534645.2011.605573

[39] Lee Humphreys. 2010. Mobile social networks and urban public space. New Media \& Society 12, 5 (Aug. 2010), 763-778. https://doi.org/10.1177/1461444809349578

[40] Lee Humphreys. 2013. Mobile social media: Future challenges and opportunities. Mobile Media \& Communication 1, 1 (Jan. 2013), 20-25. https://doi.org/10.1177/2050157912459499

[41] Lee Humphreys and Tony Liao. 2013. Foursquare and the parochialization of public space. First Monday 18, 11 (Nov. 2013). https://doi.org/10.5210/fm.v18i11.4966

[42] Catherine Jones and Konstantinos Papangelis. 2020. Reflective practice: Lessons learnt by using board games as a design tool for location-based games. In Geospatial Technologies for Local and Regional Development, Phaedon Kyr iakidis, Diofantos Hadjimitsis, Dimitrios Skarlatos, and Ali Mansourian (Eds.). Springer International Publishing, Cham, Switzerland, 291-307.

[43] M. Karrholm. 2007. The materiality of territorial production: A conceptual discussion of territoriality, materiality, and the everyday life of public space. Space and Culture 10, 4 (Nov. 2007), 437-453. https://doi.org/10.1177/ 1206331207304356

[44] Jussi Parikka. 2013. What is media archaeology? Literary and Linguistic Computing 28, 3 (Sept. 2013), 484-486. https: //doi.org/10.1093/llc/fqs061

[45] J. Richard Landis and Gary G. Koch. 1977. The measurement of observer agreement for categorical data. Biometrics 33, 1 (1977), 159-174. http://www.jstor.org/stable/2529310.

[46] Henri Lefebvre and Donald Nicholson-Smith. 2011. The Production of Space. Blackwell, Malden, MA.

[47] Paul Legris, John Ingham, and Pierre Collerette. 2003. Why do people use information technology? A critical review of the technology acceptance model. Information \& Management 40, 3 (Jan. 2003), 191-204. https://doi.org/10.1016/S03787206(01)00143-4

[48] Grant MacCracken. 1997. The Long Interview. Qualitative Research Methods Book 13. SAGE, Newbury Park, CA.

[49] Nyala Noë, Roger M. Whitaker, Martin J. Chorley, and Thomas V. Pollet. 2016. Birds of a feather locate together? Foursquare checkins and personality homophily. Computers in Human Behavior 58 (May 2016), 343-353. https://doi. org/10.1016/j.chb.2016.01.009

[50] Tomasz Oleksy and Anna Wnuk. 2017. Catch them all and increase your place attachment! The role of location-based augmented reality games in changing people-place relations. Computers in Human Behavior 76 (Nov. 2017), 3-8. https://doi.org/10.1016/j.chb.2017.06.008 
[51] Konstantinos Papangelis, Alan Chamberlain, Vassilis-Javed Khan, Ioanna Lykourentzou, Michael Saker, Hai Ning Liang, Irwyn Sadien, and Ting Cao. 2019. Performing the digital self: Understanding location-based social networking, territory, space, and identity in the city. ACM Transactions on Computer-Human Interaction 27, 1 (2019), 1-26.

[52] Konstantinos Papangelis, Alan Chamberlain, and Hai-Ning Liang. 2016. New directions for preserving intangible cultural heritage through the use of mobile technologies. In Proceedings of the 18th International Conference on HumanComputer Interaction with Mobile Devices and Services Adjunct (MobileHCI'16). ACM, New York, NY, 964-967. https: //doi.org/10.1145/2957265.2962643

[53] Konstantinos Papangelis, Melvin Metzger, Yiyeng Sheng, Hai-Ning Liang, Alan Chamberlain, and Ting Cao. 2017. Conquering the city: Understanding perceptions of mobility and human territoriality in location-based mobile games. Proceedings of the ACM on Interactive, Mobile, Wearable and Ubiquitous Technologies 1, 3 (Sept. 2017), 1-24. https: //doi.org/10.1145/3130955

[54] Konstantinos Papangelis, Melvin Metzger, Yiyang Sheng, Hai-Ning Liang, Alan Chamberlain, and Vassilis-Javed Khan. 2017. "Get off my lawn!”: Starting to understand territoriality in location based mobile games. In Proceedings of the 2017 CHI Conference Extended Abstracts on Human Factors in Computing Systems (CHI EA'17). ACM, New York, NY, 1955-1961. https://doi.org/10.1145/3027063.3053154

[55] Konstantinos Papangelis, Yiyang Sheng, Hai-Ning Liang, Alan Chamberlain, Vassilis-Javed Khan, and Ting Cao. 2017. Unfolding the interplay of self-identity and expressions of territoriality in location-based social networks. In Proceedings of the 2017 ACM International foint Conference on Pervasive and Ubiquitous Computing and Proceedings of the 2017 ACM International Symposium on Wearable Computers (UbiComp'17). ACM, New York, NY, 177-180. https://doi.org/10.1145/3123024.3123081

[56] Jiayin Qi, Emmanuel Monod, Binxing Fang, and Shichang Deng. 2018. Theories of social media: Philosophical foundations. Engineering 4, 1 (Feb. 2018), 94-102. https://doi.org/10.1016/j.eng.2018.02.009

[57] Ayalla Ruvio and Russell W. Belk (Eds.). 2013. The Routledge Companion to Identity and Consumption. Routledge, London, UK.

[58] Michael Saker. 2016. Foursquare and identity: Checking-in and presenting the self through location. New Media \& Society 19, 6 (2016), 1461444815625936. http://nms.sagepub.com/content/early/2016/03/24/1461444815625936.

[59] Michael Saker and Leighton Evans. 2016. Locative media and identity: Accumulative technologies of the self. SAGE Open 6, 3 (2016), 1-10. http://eprints.brighton.ac.uk/15910/.

[60] Michael Saker and Leighton Evans. 2016. Locative mobile media and time: Foursquare and technological memory. First Monday 21, 2 (Jan. 2016). http://ojphi.org/ojs/index.php/fm/article/view/6006.

[61] Tim Schwanen and Mei-Po Kwan. 2008. The Internet, mobile phone and space-time constraints. Geoforum 39, 3 (May 2008), 1362-1377. https://doi.org/10.1016/j.geoforum.2007.11.005

[62] Raz Schwartz and Germaine R. Halegoua. 2015. The spatial self: Location-based identity performance on social media. New Media \& Society 17, 10 (2015), 1643-1660. http://journals.sagepub.com/doi/abs/10.1177/1461444814531364.

[63] Theresa M. Senft. 2013. Microcelebrity and the branded self. In A Companion to New Media Dynamics, John Hartley, Jean Burgess, and Axel Bruns (Eds.). Wiley-Blackwell, Oxford, UK, 346-354. https://doi.org/10.1002/9781118321607. $\operatorname{ch} 22$

[64] Eugenia Siapera. 2011. Understanding New Media. SAGE, Thousand Oaks, CA.

[65] Edward W. Soja. 1996. Thirdspace: Journeys to Los Angeles and Other Real-and-Imagined Places. Blackwell, Cambridge, MA.

[66] Bernard Stiegler. 1998. Technics and Time. Stanford University Press, Stanford, CA.

[67] Daniel M. Sutko and Adriana de Souza e Silva. 2011. Location-aware mobile media and urban sociability. New Media \& Society 13, 5 (Aug. 2011), 807-823. https://doi.org/10.1177/1461444810385202

[68] Duncan J. Watts and Steven H. Strogatz. 1998. Collective dynamics of 'small-world' networks. Nature 393, 6684 (June 1998), 440-442. https://doi.org/10.1038/30918

[69] Rowan Wilken. 2008. Mobilizing place: Mobile media, peripatetics, and the renegotiation of urban places. fournal of Urban Technology 15, 3 (Dec. 2008), 39-55. https://doi.org/10.1080/10630730802677939

[70] Yu Zheng. 2011. Location-based social networks: Users. In Computing with Spatial Trajectories, Yu Zheng and Xiaofang Zhou (Eds.). Springer, New York, NY, 243-276. https://doi.org/10.1007/978-1-4614-1629-6_8

[71] John Zimmerman, Jodi Forlizzi, and Shelley Evenson. 2007. Research through design as a method for interaction design research in HCI. In Proceedings of the SIGCHI Conference on Human Factors in Computing Systems. ACM, New York, NY, 493. https://doi.org/10.1145/1240624.1240704

Received June 2020; revised June 2021; accepted June 2021 